\title{
Deformation Analysis of Matrix Models
}

\author{
John Palmer \\ Department of Mathematics \\ University of Arizona \\ Tucson, AZ 85721
}

Introduction. Suppose that $\varphi$ and $\psi$ are functions defined on the real line and let

$$
K(x, y)=\frac{\varphi(x) \psi(y)-\psi(x) \varphi(y)}{x-y} \chi(y),
$$

where $\chi$ is the characteristic function of some union of intervals

$$
J=\cup_{j=1}^{m}\left[a_{2 j-1}, a_{2 j}\right] .
$$

We regard $K(x, y)$ as the kernel of an integral operator $K$

$$
K f(x)=\int_{\mathbf{R}} K(x, y) f(y) d y .
$$

For special choices of $\varphi$ and $\psi$, Fredholm determinants of the form

$$
\operatorname{det}(1-K)
$$

arise in the theory of random Hermitian matrices [8]. In this theory it is a result of Gaudin and Mehta that such determinants give the probability that no eigenvalues for an $N \times N$ random matrix lie in the set $J$ (the limit $N \rightarrow \infty$ is also of interest in the context of various scaling limits [12]). More generally the derivative

$$
\left.\frac{(-1)^{n}}{n !} \frac{d^{n}}{d \lambda^{n}} \operatorname{det}(1-\lambda K)\right|_{\lambda=1}
$$

is the probability that exactly $n$ eigenvalues of the random matrix lie in the interval $J$.

Fredholm determinants of a similar sort arise for certain solvable models in two dimensional quantum field theory [4], [3]. In the pioneering work [4], it was shown for the simplest $N \rightarrow \infty$ limit in random matrix theory and for impenetrable Bosons in two dimensional field theory, that it is possible to express the relevant Fredholm determinant in terms of the solution to a completely integrable non-linear system of differential equations. In [3] some other field theory models are shown to have this same property. In reference to this phenomenon, Its, Izergin, Korepin, and Slavnov 
refer to kernels of type (0.1) as "completely integrable integral kernels". Recently, Tracy and Widom [15], have given a systematic treatment in the context of random matrix models. They show that if the functions $\varphi$ and $\psi$ satisfy a linear differential equation

$$
\frac{d}{d z}\left[\begin{array}{l}
\varphi \\
\psi
\end{array}\right]=\Omega(z)\left[\begin{array}{l}
\varphi \\
\psi
\end{array}\right]
$$

where

$$
\Omega(z)=\frac{1}{m(z)}\left[\begin{array}{cc}
A(z) & B(z) \\
-C(z) & -A(z)
\end{array}\right],
$$

and $m(z), A(z), B(z)$ and $C(z)$ are polynomials, then the Fredholm determinant, $\operatorname{det}(1-K)$, can be expressed in terms of the solution to a system of non linear differential equations.

In this note we will show that the differential equations found by Tracy and Widom are special cases of monodromy preserving deformation equations, and the analysis of the Fredholm determinant is a special case of the general analysis of $\tau$ - functions [5]. This is not the first time that a connection between random matrices and monodromy preserving deformation theory has been observed. Moore, [9], [10], has already observed that families of differential equations of type $(0.2)$ play a role in the analysis of the partition function for random matrix models. In his work the singularity at infinity for (0.2) is important. The deformations of the coefficients $m, A, B$ and $C$ that he considers all preserve the "Stokes' multipliers" for $(0.2)$, i.e., the monodromy data at infinity. The partition function is a $\tau$ - function for the resulting monodromy preserving deformation, and the general connection between $\tau$ - functions and non-linear equations accounts, in particular, for the expression which gives the partition function for two dimensional gravity in terms of a Painlevé transcendent. Some interesting mathematical problems that arise in Moore's analysis have been solved by Beals and Sattinger in [1]. In particular they prove that certain deformations of $(0.2)$, governed by compatible linear differential equations for $\varphi$ and $\psi$, do indeed leave the Stokes' multipliers at infinity invariant.

Here a local perturbation of (0.2) with simple poles at the points $a_{j}$ will play a central role (see (1.14) below). The compatibility of the linear differential equations (1.14) and (1.15) will lead naturally to the non-linear differential equations found by Tracy and Widom. Realizing the non-linear equations as compatibility conditions for linear systems is not new. In [2] Harnad, Tracy and Widom obtain the differential equations for the sine kernel and the Airy kernel as compatibility conditions for linear systems that are essentially the same as the linear systems we find below. Apparently, these linear systems were guessed on the basis of experience with the dynamical systems on coadjoint orbits of loop algebras that are the central theme of [2]. One advantage of our treatment is that the linear systems are very simple 
to get at and the connection with dynamical systems on coadjoint orbits of loop algebras is then direct (in the setting which is explained in [2]).

This paper is organized in two parts. In the first part we show how to realize the Tracy-Widom equations as monodromy preserving deformation equations, staying close to the integral equation which is central in their work. In the second part we introduce the formalism of Malgrange [7] in order to discuss the deformation theoretic $\tau$ - function. Up to a trivial factor we show that this is the determinant, $\operatorname{det}(1-\lambda K)$. The formalism of Malgrange is, incidently, better adapted to deal with analyticity questions in the $a_{j}$ parameters.

The author would like to thank C. Tracy and H. Widom for helpful conversations about their work. 


\section{$\S 1$ The Deformation Problem}

We will now introduce the deformation problem which will lead to the linear equations (1.14) and (1.15). We will consider the problem in light of the formalism for Cauchy-Riemann operators introduced in [11]. It is not essential to understand the formalism in [11] to follow what goes on but it does provide some motivation. Suppose to begin that

$$
\left[\begin{array}{l}
\varphi(z) \\
\psi(z)
\end{array}\right]
$$

is a solution to the differential equation (0.2) defined for $z \in \mathbf{C}$, the complex plane. Here $m, A, B$, and $C$ are assumed to be polynomials as mentioned above. In case $m$ is a non constant polynomial it may happen that the differential equation (0.2) does not have any globally defined single valued solutions. To avoid this complication at the start we will suppose that $m=1$. Let

$$
\left[\begin{array}{l}
\widetilde{\varphi}(z) \\
\widetilde{\psi}(z)
\end{array}\right]
$$

be a second independent solution to the same differential equation (0.2). Since the trace of the matrix on the right hand side of $(0.2)$ is 0 the determinant of the fundamental solution

$$
\left[\begin{array}{ll}
\varphi & \widetilde{\varphi} \\
\psi & \widetilde{\psi}
\end{array}\right]
$$

is constant and we can choose $\widetilde{\varphi}$ and $\widetilde{\psi}$ so that this determinant is 1 . We wish to consider the Cauchy Riemann operator

$$
\left[\begin{array}{cc}
\varphi & \widetilde{\varphi} \\
\psi & \widetilde{\psi}
\end{array}\right]^{-1}\left[\begin{array}{cc}
\bar{\partial}_{z} & 0 \\
0 & \bar{\partial}_{z}
\end{array}\right]\left[\begin{array}{cc}
\varphi & \widetilde{\varphi} \\
\psi & \widetilde{\psi}
\end{array}\right]
$$

In this formula $\bar{\partial}_{z}=\frac{1}{2}\left(\partial_{x}+i \partial_{y}\right)$ as usual. Of course, since $\varphi, \widetilde{\varphi}, \psi$, and $\widetilde{\psi}$ are analytic functions in $\mathbf{C}$ this $\mathrm{C}-\mathrm{R}$ operator acts precisely as the standard C-R operator

$$
\left[\begin{array}{cc}
\bar{\partial}_{z} & 0 \\
0 & \bar{\partial}_{z}
\end{array}\right]
$$

The difference is that we will take the domain of (1.2) to be the Sobolev space $H^{1}(\mathbf{C})$ so that (1.1) represents an operator with with domain

$$
\left[\begin{array}{cc}
\varphi & \widetilde{\varphi} \\
\psi & \widetilde{\psi}
\end{array}\right]^{-1} H^{1}(\mathbf{C})
$$


Note that since the determinant of the fundamental solution is 1 the inverse

$$
\left[\begin{array}{cc}
\varphi & \widetilde{\varphi} \\
\psi & \widetilde{\psi}
\end{array}\right]^{-1}=\left[\begin{array}{cc}
\widetilde{\psi} & -\widetilde{\varphi} \\
-\psi & \varphi
\end{array}\right] .
$$

Next we wish to introduce a different domain for the C-R operator (1.1) which incorporates functions with fixed branching behavior along an interval $\left[a_{1}, a_{2}\right]$ on the real axis. It will be obvious how to incorporate more than one interval $\left[a_{j}, a_{j+1}\right]$ in the formalism so I will confine my attention to just one interval for simplicity. We wish to include in the domain of our new C-R operator functions which change by a factor of

$$
M=\left[\begin{array}{cc}
1 & 2 \pi i \lambda \\
0 & 1
\end{array}\right]
$$

in a counterclockwise circuit of the left endpoint, $a_{1}$, and by the inverse of this matrix in a counterclockwise circuit of the right endpoint, $a_{2}$. To understand the resulting C-R operator we would like to realize it as a similarity transform

$$
Y^{-1}\left[\begin{array}{cc}
\bar{\partial}_{z} & 0 \\
0 & \bar{\partial}_{z}
\end{array}\right] Y
$$

of the standard C-R operator by a multivalued analytic function $Y(z)$. So that the domain of this operator is the same as the domain of (1.1) near infinity we want

$$
Y(z) \sim F(z) \text { for } z \text { near } \infty,
$$

where we write

$$
F(z)=\left[\begin{array}{ll}
\varphi(z) & \widetilde{\varphi}(z) \\
\psi(z) & \widetilde{\psi}(z)
\end{array}\right] .
$$

To find $Y(z)$ we split the problem into two pieces. Let $Y_{+}(z)=Y(z)$ for $z \in\{z$ : $\left.z_{2} \geq 0\right\}$ and $Y_{-}(z)=Y(z)$ for $z \in\left\{z: z_{2} \leq 0\right\}$. Then a more precise formulation of (1.3) is that

$$
F Y_{ \pm}^{-1}-\left[\begin{array}{ll}
1 & 0 \\
0 & 1
\end{array}\right] \in H_{ \pm}
$$

where $H_{ \pm}$is the usual Hardy space for the upper $(+)$and lower $(-)$half planes. On the real axis the boundary values $Y_{+}$and $Y_{-}$are related by

$$
Y_{+}=Y_{-}((I-\chi)+\chi M)
$$

where $\chi=\chi_{\left[a_{1}, a_{2}\right]}$ is the characteristic function of the interval. In order that the integral operator $K$ (rather than its transpose) arises in the fundamental integral 
equation (1.6) below it will be convenient to temporarily work with $Y_{ \pm}^{-1}$. The monodromy condition relating the boundary values of $Y_{ \pm}^{-1}$ becomes

$$
Y_{-}^{-1}=((I-\chi)+\chi M) Y_{+}^{-1}
$$

We can rewrite this last equation as

$$
Y_{-}^{-1}-F^{-1}=Y_{+}^{-1}-F^{-1}+\chi(M-I) Y_{+}^{-1}
$$

Let $P_{+}$be the usual orthogonal projection on $H_{+}$and define

$$
P_{+}^{F}=F^{-1} P_{+} F
$$

Then $P_{+}^{F}$ is the analogue of $P_{+}$for the $\mathrm{C}-\mathrm{R}$ operator $(1.1)$. We can write (1.4) as

$$
\begin{aligned}
& P_{+}^{F}\left(Y_{-}^{-1}-F^{-1}\right)=0, \\
& P_{+}^{F}\left(Y_{+}^{-1}-F^{-1}\right)=Y_{+}^{-1}-F^{-1},
\end{aligned}
$$

where we are not multiplying operators anymore but just applying $P_{+}^{F}$ to the columns of the matrices $Y_{ \pm}^{-1}-F^{-1}$. Applying $P_{+}^{F}$ to the columns of (1.5) and making use of these last relations one finds

$$
Y_{+}^{-1}+P_{+}^{F} \chi(M-I) Y_{+}^{-1}=F^{-1} .
$$

Since the kernel of $P_{+}$is $-\frac{1}{2 \pi i}(x-y)^{-1}$ (with $x=x+i 0$ ) we find that the kernel of the operator $P_{+}^{F} \chi(M-I)$ is

$$
-\frac{\lambda \chi(y)}{x-y}\left[\begin{array}{cc}
0 & \widetilde{\psi}(x) \varphi(y)-\widetilde{\varphi}(x) \psi(y) \\
0 & \varphi(x) \psi(y)-\psi(x) \varphi(y)
\end{array}\right]
$$

Now let $K$ denote the operator with kernel

$$
\frac{\chi(y)}{x-y}(\varphi(x) \psi(y)-\psi(x) \varphi(y))
$$

This is a non-singular kernel. Let $\widetilde{K}$ denote the operator with kernel

$$
\frac{\chi(y)}{x-y}(\widetilde{\psi}(x) \varphi(y)-\widetilde{\varphi}(x) \psi(y))
$$


This is a singular kernel. Because of the connection with $P_{+}$it is understood that the variable $x$ should be allowed to approach the real axis from above (i.e., $x+i 0$ ). In terms of $K$ and $\widetilde{K}$ the equation (1.6) becomes

$$
\left[\begin{array}{cc}
1 & -\lambda \widetilde{K} \\
0 & 1-\lambda K
\end{array}\right] Y_{+}^{-1}=F^{-1} .
$$

This is solvable provided $1-\lambda K$ is invertible. We suppose that this is the case in what follows. As we will see in the second section, the $\tau$-function for this problem is just $\operatorname{det}(1-\lambda K)$.

Solving equation (1.7) for $Y_{+}^{-1}$ one finds

$$
Y_{+}^{-1}=\left[\begin{array}{cc}
\widetilde{\psi}-\lambda \widetilde{K} P & -\widetilde{\varphi}+\lambda \widetilde{K} Q \\
-P & Q
\end{array}\right],
$$

where

$$
\begin{aligned}
& Q=(1-\lambda K)^{-1} \varphi, \\
& P=(1-\lambda K)^{-1} \psi .
\end{aligned}
$$

We have here adopted the notation from [15] to facilitate comparisons. From the integral equation

$$
F Y_{+}^{-1}=I-P_{+}\left(F \chi(M-I) Y_{+}^{-1}\right),
$$

it is clear that $F Y_{+}^{-1}$ has an analytic continuation into the upper half plane. Since $F$ is analytic and invertible in the whole plane, $Y_{+}$will be analytic in the upper half plane. The same argument shows that $Y_{-}$has an analytic continuation into the lower half plane. The two functions $Y_{+}$and $Y_{-}$fit together to give a single analytic function $Y(z)$ which has a branch cut joining $a_{1}$ and $a_{2}$. We want to analyse the singularities of $Y(z)$ in a neighborhood of the points $a_{j}$ and near $\infty$. First we consider the local singularity at $a_{1}$. Define $f(z)=\log \left(z-a_{1}\right)$, with the branch cut for the logarithm taken to be the ray $\left[a_{1}, \infty\right)$. Apart from the location of the branch cut it does not matter which determination of the logarithm we use. We claim that the matrix

$$
Y(z)\left[\begin{array}{cc}
1 & \lambda f(z) \\
0 & 1
\end{array}\right]=G(z),
$$

is single valued and analytic in a neighborhood of $z=a_{1}$. The left hand side of (1.9) is single valued because the monodromy of the matrix

$$
\left[\begin{array}{cc}
1 & \lambda f(z) \\
0 & 1
\end{array}\right]
$$

as $z$ makes a circuit of $a_{1}$ exactly cancels the monodromy that is built into $Y(z)$ by construction. The left hand side of (1.9) will have a removable singularity at $a_{1}$ 
because $P(z)$ and $Q(z)$ are regular at $z=a_{1}$ and $\widetilde{K}$ introduces only a logarithmic singularity at $z=a_{1}$.

Taking determinants of both sides of (1.9) shows that $\operatorname{det}(Y(z))$ is an analytic function of $z$ near $z=a_{1}$. In a similar fashion one can $\operatorname{show}$ that $\operatorname{det}(Y(z))$ is analytic near $z=a_{2}$. From (1.8) it follows that

$$
\operatorname{det}\left(F^{-1} Y(z)\right)=\operatorname{det}(Y(z))=1+O\left(z^{-1}\right),
$$

for $z$ in a neighborhood of $\infty$. Thus $\operatorname{det}(Y(z))=1$ for all $z$. It follows from this and the earlier expression for $Y$ that

$$
Y=\left[\begin{array}{ll}
Q & \widetilde{\varphi}-\lambda \widetilde{K} Q \\
P & \widetilde{\psi}-\lambda \widetilde{K} P
\end{array}\right]
$$

which displays the analogous roles played by $\varphi$, and $\psi$ and $Q$, and $P$.

Differentiating (1.9) with respect to $z$ one finds

$$
\frac{\lambda Y(z)}{z-a_{1}}\left[\begin{array}{ll}
0 & 1 \\
0 & 0
\end{array}\right]+Y^{\prime}(z)\left[\begin{array}{cc}
1 & \lambda f(z) \\
0 & 1
\end{array}\right]=G^{\prime}(z)
$$

which may be easily rewritten

$$
Y^{\prime}(z) Y^{-1}(z)=-\frac{\lambda}{z-a_{1}} Y(z)\left[\begin{array}{cc}
0 & 1 \\
0 & 0
\end{array}\right] Y^{-1}(z)+G^{\prime}(z) G^{-1}(z)
$$

Now using the fact that the determinant of $Y$ is 1 and the formula for $Y$ in terms of $P$ and $Q$ above one finds that

$$
Y^{\prime}(z) Y^{-1}(z)=\frac{\lambda}{z-a_{1}}\left[\begin{array}{cc}
q_{1} p_{1} & -q_{1}^{2} \\
p_{1}^{2} & -q_{1} p_{1}
\end{array}\right]+O(1) \text { for } z \sim a_{1}
$$

In this formula we've written $q_{j}=Q\left(a_{j}\right)$ and $p_{j}=P\left(a_{j}\right)$. In a precisely analogous fashion we calculate the residue of $Y^{\prime} Y^{-1}$ at $z=a_{2}$ to find

$$
Y^{\prime}(z) Y^{-1}(z)=-\frac{\lambda}{z-a_{2}}\left[\begin{array}{cc}
q_{2} p_{2} & -q_{2}^{2} \\
p_{2}^{2} & -q_{2} p_{2}
\end{array}\right]+O(1) \text { for } z \sim a_{2} .
$$

Now define

$$
\Delta=-P_{+} F(M-I) \chi Y_{+}^{-1}
$$

Differentiating (1.8) with respect to $z$ one finds

$$
F^{\prime} Y^{-1}-F Y^{-1} Y^{\prime} Y^{-1}=\Delta^{\prime}
$$


or

$$
F^{\prime} F^{-1}\left(F Y^{-1}\right)-\left(F Y^{-1}\right) Y^{\prime} Y^{-1}=\Delta^{\prime}
$$

Since $F Y^{-1}=I+\Delta$ this can be written

$$
Y^{\prime} Y^{-1}=(I+\Delta)^{-1} \Omega(I+\Delta)-(I+\Delta)^{-1} \Delta^{\prime}
$$

Since $\Delta(z)=O\left(z^{-1}\right)$ and $\Delta^{\prime}(z)=O\left(z^{-2}\right)$ it follows that the principal part of $Y^{\prime} Y^{-1}$ at $\infty$ is the same as the principal part of

$$
(I+\Delta)^{-1} \Omega(I+\Delta) \text {. }
$$

It now follows from this observation and (1.11) and (1.12) that there is a matrix valued polynomial $M(z)$ of the same degree as $\Omega(z)$ so that

$$
\frac{d Y}{d z} Y^{-1}=M(z)+\frac{\lambda C_{1}}{z-a_{1}}-\frac{\lambda C_{2}}{z-a_{2}}
$$

where

$$
C_{k}=\left[\begin{array}{cc}
q_{k} p_{k} & -q_{k}^{2} \\
p_{k}^{2} & -q_{k} p_{k}
\end{array}\right] .
$$

Now we determine the differential equation satisfied by $Y$ in the $a_{j}$ variables. Differentiating (1.8) in the $a$ variables one finds

$$
d_{a} Y Y^{-1}=O\left(z^{-1}\right) \text { as } z \rightarrow \infty
$$

Differentiating (1.9) in the $a$ variables one finds the principal part of $d_{a} Y Y^{-1}$ at $a_{j}$ is:

$$
(-1)^{k} \frac{\lambda C_{k}}{z-a_{k}} d a_{k}
$$

Combining these two observations one finds that the function $d_{a} Y Y^{-1}$ is analytic in $z$ on $\mathbf{P}^{1}$ except for simple poles at $a_{1}$ and $a_{2}$. It has a zero at $z=\infty$ and so

$$
d_{a} Y Y^{-1}=-\frac{\lambda C_{1}}{z-a_{1}} d a_{1}+\frac{\lambda C_{2}}{z-a_{2}} d a_{2} .
$$

Now we apply these observations to deduce non-linear deformation equations. If one examines the equality of mixed partials

$$
\frac{\partial}{\partial a_{j}} \frac{\partial}{\partial z} Y=\frac{\partial}{\partial z} \frac{\partial}{\partial a_{j}} Y
$$


using (1.14) and (1.15), one obtains an equation relating rational functions of $z$ with poles at $a_{j}$ and $\infty$. Equating the principal parts at the finite poles $a_{j}$, one finds

$$
\begin{aligned}
& d_{a} C_{1}=\left[M\left(a_{1}\right), C_{1}\right] d a_{1}+\lambda\left[C_{1}, C_{2}\right] \frac{d\left(a_{2}-a_{1}\right)}{a_{2}-a_{1}} \\
& d_{a} C_{2}=\left[M\left(a_{2}\right), C_{2}\right] d a_{2}+\lambda\left[C_{1}, C_{2}\right] \frac{d\left(a_{2}-a_{1}\right)}{a_{2}-a_{1}}
\end{aligned}
$$

where $M\left(a_{j}\right)$ is the value of the matrix polynomial $M(z)$ at $z=a_{j}$. Equating the principal parts at $\infty$ one finds

$$
\frac{\partial M(z)}{\partial a_{j}}=(-1)^{j} \lambda\left[\frac{M(z)-M\left(a_{j}\right)}{z-a_{j}}, C_{j}\right] .
$$

Note that in this last equation each side is a polynomial function of $z$ of order 1 less than the degree, $n$, of $M(z)$ (since the highest order term in $M(z)$ is clearly independent of $a_{j}$ ). In particular if we write

$$
M(z)=M_{n} z^{n}+M_{n-1} z^{n-1}+\ldots
$$

then it is easy to see that (1.17) implies

$$
\frac{\partial M_{n-1}}{\partial a_{j}}=(-1)^{j} \lambda\left[M_{n}, C_{j}\right]
$$

In order to make (1.16) and (1.17) more explicit we must use (1.13) to compute the polynomial $M(z)$. To make use of (1.13) we must in turn calculate the asymptotics of $\Delta(z)$ at $z=\infty$. Substituting for $Y_{+}$and $F$ in the formula for $\Delta(z)$ one finds

$$
\Delta(z)=\int_{\mathbf{R}} \frac{\lambda \chi(y)}{z-y}\left[\begin{array}{ll}
-\varphi(y) P(y) & \varphi(y) Q(y) \\
-\psi(y) P(y) & \psi(y) Q(y)
\end{array}\right] d y
$$

Expanding the denominator in a geometric series

$$
\frac{1}{z-y}=\frac{1}{z}\left(1+\frac{y}{z}+\left(\frac{y}{z}\right)^{2}+\cdots\right)
$$

one finds that

$$
\Delta(z)=\frac{\lambda}{z}\left[\begin{array}{ll}
-\widetilde{v}_{0} & u_{0} \\
-w_{0} & v_{0}
\end{array}\right]+\frac{\lambda}{z^{2}}\left[\begin{array}{ll}
-\widetilde{v}_{1} & u_{1} \\
-w_{1} & v_{1}
\end{array}\right]+O\left(z^{-3}\right)
$$


where

$$
\begin{aligned}
u_{j} & =\int_{\mathbf{R}} \chi(y) y^{j} \varphi(y) Q(y) d y, \\
\widetilde{v}_{j} & =\int_{\mathbf{R}} \chi(y) y^{j} \varphi(y) P(y) d y, \\
v_{j} & =\int_{\mathbf{R}} \chi(y) y^{j} \psi(y) Q(y) d y, \\
w_{j} & =\int_{\mathbf{R}} \chi(y) y^{j} \psi(y) P(y) d y,
\end{aligned}
$$

and the notation is again taken from [15]. We use (1.18) and (1.13) to calculate the matrix $M(z)$ in a few of the cases considered in [15].

Our first example is the sine kernel with

$$
\begin{aligned}
& \varphi(z)=\sin (z), \\
& \psi(z)=\cos (z) .
\end{aligned}
$$

In this case

$$
\Omega(z)=\left[\begin{array}{cc}
0 & 1 \\
-1 & 0
\end{array}\right],
$$

and a simple calculation shows that

$$
M_{\text {Sine }}(z)=\left[\begin{array}{cc}
0 & 1 \\
-1 & 0
\end{array}\right] \text {. }
$$

Our second example is the Airy kernel with

$$
\begin{aligned}
& \varphi(z)=\operatorname{Ai}(z), \\
& \psi(z)=\operatorname{Ai}^{\prime}(z) .
\end{aligned}
$$

In this case

$$
\Omega(z)=\left[\begin{array}{ll}
0 & 1 \\
z & 0
\end{array}\right] .
$$

Without difficulty one may use (1.13) and (1.18) to compute

$$
M_{\text {Airy }}(z)=\left[\begin{array}{cc}
-\lambda u & 1 \\
z-\lambda(v+\widetilde{v}) & \lambda u
\end{array}\right]
$$

where we've written $u=u_{0}, v=v_{0}$ and $\widetilde{v}=\widetilde{v}_{0}$. For $\lambda=1$ this simplifies somewhat since $v_{0}=\widetilde{v}_{0}$ in this event. 
Our last example is one that arises in 2D quantum gravity matrix models (the case of pure gravity). A function $\xi(x)$ is given which satisfies the differential equation

$$
\xi^{\prime \prime \prime}(x)+6 \xi(x) \xi^{\prime}(x)+4=0,
$$

which is a special case of the string equation. The functions $\varphi(z, x)$ and $\psi(z, x)$ now depend on the parameter $x$ and satisfy the differential equation

$$
\frac{d}{d z}\left[\begin{array}{l}
\varphi \\
\psi
\end{array}\right]=\Omega(z)\left[\begin{array}{l}
\varphi \\
\psi
\end{array}\right]
$$

where

$$
\Omega(z)=\left[\begin{array}{cc}
-\frac{1}{4} \xi^{\prime}(x) & z+\frac{1}{2} \xi(x) \\
-z^{2}-\frac{1}{2} \xi(x) z-\frac{1}{2} \xi^{2}(x)-\frac{1}{4} \xi^{\prime \prime}(x) & \frac{1}{4} \xi^{\prime}(x)
\end{array}\right] .
$$

This is incidently, an example of a family of differential equations in which the Stokes' multipliers at $\infty$ are independent of $x$ [9], [10]. Again one may use (1.13) and (1.18) to compute

$$
M_{\text {Gravity }}(z)=\Omega(z)+M_{1} z+M_{0}
$$

where

$$
M_{1}=\left[\begin{array}{cc}
\lambda u & 1 \\
\lambda(v+\widetilde{v}) & -\lambda u
\end{array}\right]
$$

and

$$
M_{0}=\lambda\left[\begin{array}{cc}
-\lambda u v+\frac{1}{2} \xi(x) u-w+u_{1} & \lambda u^{2}+(v+\widetilde{v}) \\
\lambda(u w-v(v+\widetilde{v}))+\xi(x) v+\widetilde{v}_{1}+v_{1} & \lambda u v-\frac{1}{2} \xi(x) u+w-u_{1}
\end{array}\right]
$$

where we've written $u=u_{0}, v=v_{0}$ and $\widetilde{v}=\widetilde{v}_{0}$.

It is straightforward to use these results to determine the differential equations (1.16) and (1.17). We will not do this here but instead we will refer the reader to [15], where the non-linear equations for $q_{j}, p_{j}, u_{j}, \ldots$ are written out, and some interesting examples beyond those mentioned above are considered. Tracy and Widom also show that equations (1.16) and (1.17) sometimes have additional first integrals which can be used to reduce the number of variables in the deformation equations. In several examples they show that the the deformation equations can be integrated in terms of Painlevé transcendents (thus generalizing the original observation in [4] that Painlevé $\mathrm{V}$ transcendents arise in the integration of the deformation equations associated with the sine kernel).

To conclude this section we will describe the changes which need to be made when the differential equation for $\varphi$ and $\psi$ already has poles in the finite plane. The 
simplest example of this sort considered by Tracy and Widom is the Bessel kernel, for which

$$
\begin{aligned}
& \varphi(z)=J_{\alpha}(\sqrt{z}), \\
& \psi(z)=z J_{\alpha}^{\prime}(\sqrt{z}) .
\end{aligned}
$$

One can see from this that $\varphi(z)$ and $\psi(z)$ will not be single valued in the complex plane. One can deal with this situation by introducing a branch cut for $\varphi$ and $\psi$ joining 0 to $\infty$. Since the intervals $\left[a_{j}, a_{j+1}\right]$ in this model will all be subsets of the positive real line it is convenient, in this case, to choose the branch cut on the negative real axis. The differential equation satisfied by $\varphi$ and $\psi$ is

$$
\frac{d}{d z}\left[\begin{array}{l}
\varphi \\
\psi
\end{array}\right]=\frac{1}{z}\left[\begin{array}{cc}
0 & 1 \\
\frac{1}{4}\left(\alpha^{2}-z\right) & 0
\end{array}\right]\left[\begin{array}{l}
\varphi \\
\psi
\end{array}\right],
$$

which has a simple pole at 0 in addition to an irregular singular point at infinity. Extending $\left[\begin{array}{l}\varphi \\ \psi\end{array}\right]$ to a matrix valued fundamental solution $F$ with determinant 1 is possible as before provided one admits a branch cut for $F$ on the negative real axis. Let $F_{+}$denote the analytic function $F$ in the upper half plane and let $F_{-}$denote the analytic function $F$ in the lower half plane. The boundary values of these two functions on the real axis are related by

$$
F_{+}=F_{-}\left(I+\chi_{0}\left(M_{0}-I\right)\right)
$$

where $\chi_{0}$ is the characteristic function of the interval $(-\infty, 0]$ and $M_{0}$ is the monodromy matrix associated with the fundamental solution $F$. We will want the monodromy of the fundamental solution $Y$ to match that of $F$ on the negative real real axis and so the relation we desire between the boundary values $Y_{ \pm}$is

$$
Y_{-}^{-1}=\left(I+\chi_{0}\left(M_{0}-I\right)+\chi(M-I)\right) Y_{+}^{-1}
$$

Using (1.22) to write $F_{-}$in terms of $F_{+}$, and the fact that $\chi_{0} \chi=0$, one can multiply (1.23) on the left by $F_{-}$to get

$$
F_{-} Y_{-}^{-1}-I=F_{+} Y_{+}^{-1}-I+F_{+} \chi(M-I) Y_{+}^{-1}
$$

If we now insist that

$$
P_{ \pm}\left(F_{ \pm} Y_{ \pm}^{-1}-I\right)=F_{ \pm} Y_{ \pm}^{-1}-I
$$

as before, then we find

$$
F_{+} Y_{+}^{-1}=I-P_{+} F_{+}(M-I) \chi Y_{+}^{-1}
$$


instead of (1.8). Now let

$$
\Delta=-P_{+} F(M-I) \chi Y_{+}^{-1}
$$

and differentiate (1.24) with respect to $z$ to obtain

$$
Y_{+}^{\prime} Y_{+}^{-1}=(I+\Delta)^{-1} \Omega(I+\Delta)-(I+\Delta)^{-1} \Delta^{\prime} .
$$

Now since $\Delta(z)=O\left(z^{-1}\right)$ and $\Delta^{\prime}(z)=O\left(z^{-2}\right)$ we can conclude that the principal part of $z Y_{+}^{\prime}(z) Y_{+}(z)^{-1}$ at $z=\infty$ is the same as the principal part of

$$
(I+\Delta(z))^{-1} z \Omega(z)(I+\Delta(z)) .
$$

The calculation of the principal part of (1.25) at infinity simultaneously determines the residues for $Y^{\prime} Y^{-1}$ at $\infty$ and at 0 . The singularity analysis of $Y^{\prime} Y^{-1}$ near $a_{j}$ for $j=1,2$ is unchanged from before and one finds

$$
Y^{\prime} Y^{-1}=-\frac{1}{4}\left[\begin{array}{ll}
0 & 0 \\
1 & 0
\end{array}\right]+\frac{\lambda}{4 z}\left[\begin{array}{cc}
u & 4 \\
\alpha^{2}+v+\widetilde{v} & -u
\end{array}\right]+\frac{\lambda C_{1}}{z-a_{1}}-\frac{\lambda C_{2}}{z-a_{2}}
$$

where we've written $u=u_{0}, v=v_{0}$ and $\widetilde{v}=\widetilde{v}_{0}$. The analysis of the singularities in $d_{a} Y Y^{-1}$ proceeds as above and we find (1.15) is unchanged. The non-linear equations are deduced as compatibility conditions for (1.26) and (1.15). Once again we refer the reader to [14] and [15] for a more detailed look at the resulting equations.

\section{$\S 2$ The tau function}

In this section we will show that the determinant $\operatorname{det}(1-\lambda K)$ which is the focal point of the random matrix applications is the $\tau$ - function for a deformation problem. As in the first section there is no serious loss of generality in considering the case of just a single interval $\left(a_{1}, a_{2}\right)$ and so for simplicity we shall do so. It is also simpler to discuss the case in which $m(z)=1$, and although it is not essential to do so, we will confine our attention in $\S 2$ to this special case.

We begin by reminding the reader of a formula for the logarithmic derivative of $\operatorname{det}(1-\lambda K)$ which can be found in [15],

$$
d_{a} \log \operatorname{det}(1-\lambda K)=R\left(a_{1}, a_{1}\right) d a_{1}-R\left(a_{2}, a_{2}\right) d a_{2} .
$$

In this formula the terms $R\left(a_{j}, a_{j}\right)$ are the diagonal values for the kernel of the resolvent operator, $\lambda K(1-\lambda K)^{-1}$, for $\lambda K$. In [15] it is shown that

$$
R\left(a_{k}, a_{k}\right)=p_{k} \frac{\partial q_{k}}{\partial a_{k}}-q_{k} \frac{\partial p_{k}}{\partial a_{k}}
$$


where the notation

$$
q_{k}=Q\left(a_{k}\right), p_{k}=P\left(a_{k}\right)
$$

is as in $\S 1$. We write

$$
M(z)=\left[\begin{array}{ll}
M_{11}(z) & M_{12}(z) \\
M_{21}(z) & M_{22}(z)
\end{array}\right]
$$

in (1.16) and find, using (2.1), that,

$$
R\left(a_{k}, a_{k}\right)=\operatorname{Tr}\left(M\left(a_{k}\right) C_{k}\right)+(-1)^{k-1} \lambda \frac{\operatorname{Tr}\left(C_{1} C_{2}\right)}{a_{2}-a_{1}} .
$$

We write $\operatorname{Tr}$ to denote the trace of a matrix. The notation $M\left(a_{j}\right)$ is slightly misleading since $M(z)$ is a polynomial in $z$ with coefficients that are functions of $a=\left(a_{1}, a_{2}\right)$. To make the dependence on the point $a$ a little clearer we should write $M(z, a)$ for $M(z)$. Then $M\left(a_{j}\right)=M\left(a_{j}, a\right)$. We will use both notations depending on circumstances.

Using (2.0) and (2.2) we will make contact with the formula for the log derivative of the tau function. In [5] Jimbo, Miwa and Ueno have given a formula for the log derivative of the tau function in the general case of irregular singular points. In [7] Malgrange has provided a geometrical significance for the tau function in the case of regular singular points with simple poles. Instead of simply adopting the JMU formula we will introduce the slight modifications in Malgrange's analysis that are necessary to deal with the irregular singular point at infinity in (1.14). We hope that this will prove to be more instructive.

First, fix points $a_{j}^{0} \in \mathbf{R} \subset \mathbf{C}$ which are distinct for $j=1,2$. We suppose that the integral operator $1-K_{0}$ is invertible. Here $K_{0}$ is the integral operator with kernel given by $(0.1)$ and the interval $J=\left(a_{1}^{0}, a_{2}^{0}\right)$. Since $1-K_{0}$ is invertible and $K_{0}$ is compact it is clear that $1-\lambda K_{0}$ will remain invertible provided that $\lambda \in \mathbf{C}$ is chosen in a sufficient small neighborhood of 1 . Throughout this section we will suppose that $\lambda$ is chosen sufficiently close to 1 .

We may rephrase the existence of $Y\left(z, a^{0}\right)$ satisfying (1.14) in the following terms. There exists a holomorphic connection $\nabla^{0}$ on the trivial bundle $\mathbf{C} \backslash\left\{a_{1}^{0}, a_{2}^{0}\right\} \times$ $\mathbf{C}^{2}$ with simple poles at $a_{1}^{0}$ and $a_{2}^{0}$ given by,

$$
\nabla^{0}=d_{z}-\Omega\left(z, a^{0}\right) d z
$$

where,

$$
\Omega(z, a)=M(z, a)+\frac{\lambda C_{1}(a)}{z-a_{1}}-\frac{\lambda C_{2}(a)}{z-a_{2}}
$$

and,

$$
d_{z}=d z \partial_{z}+d \bar{z} \bar{\partial}_{z}
$$


Note that $\nabla^{0}$ is an integrable connection (it has curvature 0) since

$$
\frac{\partial}{\partial \bar{z}} \Omega\left(z, a^{0}\right)=0
$$

for $z \in \mathbf{C} \backslash\left\{a_{1}^{0}, a_{2}^{0}\right\}$, and locally flat sections for $\nabla^{0}$ are $\mathbf{C}^{2}$ valued holomorphic functions, $s(z)$, satisfying the differential equation

$$
s^{\prime}(z)=\Omega\left(z, a^{0}\right) s(z) .
$$

One problem considered by Malgrange in [7] is the problem of deforming the connection $\nabla^{0}$ in the " $a$ " variables so that the resulting connection, $\nabla$, has simple poles at $a_{j}$ for $j=1,2$ and so that the holonomy of the resulting connection about the singular points $a_{j}$ remains fixed as the points $a_{j}$ vary. Actually, to fix the determination of holonomy for a connection we must first choose a base point, $z_{0}$, and closed simple paths, $\gamma_{j}$, about the singular points $a_{j}$. Parallel translation around the curves $\gamma_{j}$ then depends only on the homotopy class of the path $\gamma_{j}$ in $\mathbf{C} \backslash\left\{a_{1}, a_{2}\right\}$. Parallel translation about a closed curve is given by a linear transformation on the fiber over the endpoint which our fixed trivialization identifies with $\mathbf{C}^{2}$. One might consider the problem of deforming the connection $\nabla^{0}$ while keeping the holonomy matrices associated with the curves $\gamma_{j}$ fixed. However, to make contact with the work we did in the first section this is not exactly the problem we will consider. Our principal difficulty is that we would like to set $z_{0}=\infty \in \mathbf{P}^{1}$, but this is not a good choice as a base point since the point at infinity is a singular point for the connection $\nabla$. Instead of fixing the holonomy of the connection $\nabla$ we will deform $\nabla^{0}$ so that certain monodromy matrices for a distinguished fundamental solution $Y(z, a)$ to,

$$
d_{z} Y(z, a)-\Omega(z, a) Y(z, a) d z=0
$$

remain fixed as the points $a_{1}$ and $a_{2}$ vary in a neighborhood of $a_{1}^{0}$ and $a_{2}^{0}$. The distinguished fundamental solution will arise in the course of fixing the behavior of the connection $\nabla$ in a neighborhood of $\infty$. Recall that our starting point in $\S 1$ was a fundamental solution $F(z)$ to

$$
d_{z} F-\Omega(z) F d z=0
$$

where $\Omega(z)$ is given by (0.3). We will fix the behavior of $\nabla$ in a neighborhood of infinity by requiring that it be gauge equivalent to the connection,

$$
d_{z}-\Omega(z) d z
$$

in such a neighborhood, by a gauge transformation which is $I+O\left(z^{-1}\right)$ near infinity. The fundamental solution $Y(z, a)$ will be uniquely determined by the requirement that,

$$
F(z) Y(z, a)^{-1}=I+O\left(z^{-1}\right)
$$


for $z$ in a neighborhood of $\infty$. The monodromy multipliers for $Y(z, a)$ obtained by making counterclockwise circuits of $a_{1}$ and $a_{2}$ will play the role of fixed holonomy. Analytic continuation of $Y(z, a)$ along a small counterclockwise circuit of $a_{1}$ will transform $Y(z, a)$ into $Y(z, a) M$, where as in $\S 1$,

$$
M=\left[\begin{array}{cc}
1 & 2 \pi i \lambda \\
0 & 1
\end{array}\right] .
$$

Similarly, analytic continuation of $Y(z, a)$ in a small counterclockwise circuit of $a_{2}$ will transform $Y(z, a)$ into $Y(z, a) M^{-1}$.

We will next sketch the existence theory for the deformation $\nabla$ by following the arguments in Malgrange [7]. In fact, again following [7], it will be convenient to do a little more. It is possible to prove the existence of a prolongation of the connection $\nabla^{0}$ to a connection in both the $z$ and the $a$ variables in such a fashion as to incorporate (1.15) as well as (1.14).

Let $D_{j}(r)$ denote the open disk of radius $r>0$ about the point $a_{j}^{0}$ and write $\Gamma_{j}(r)$ for the circle of radius $r$ about the point $a_{j}^{0}$. We suppose that for $j=1,2$ real numbers $\rho_{1, j}, \rho_{2, j}$, and $r_{j}$ are chosen so that

$$
\rho_{2, j}>r_{j}>\rho_{1, j}>0
$$

and the numbers $\rho_{2, j}$ are small enough so that the closed disks $\bar{D}_{1}\left(\rho_{2,1}\right)$ and $\bar{D}_{2}\left(\rho_{2,2}\right)$ do not intersect. We write,

$$
\begin{aligned}
D_{j} & =D_{j}\left(\rho_{2, j}\right), \\
\Gamma_{j} & =\Gamma_{j}\left(r_{j}\right), \\
\delta D_{j} & =D_{j} \backslash \bar{D}_{j}\left(\rho_{1, j}\right) .
\end{aligned}
$$

Choose $\epsilon>0$ so that $\epsilon<\rho_{1, j}$ for $j=1,2$ and let $U$ denote the open ball of radius $\epsilon$ about the point $\left(a_{1}, a_{2}\right)$ in $\mathbf{C}^{2}$. Let

$$
\Delta_{j}=\left\{(z, a) \in D_{j} \times U \mid z=a_{j}\right\} .
$$

We pull back the connection one form $\Omega\left(z, a^{0}\right) d z$ to a connection one form, $\Omega_{j} d(z-$ $a_{j}$ ) on $D_{j} \times U \backslash \Delta_{j}$ by the translation

$$
(z, a) \rightarrow z-a_{j}+a_{j}^{0} .
$$

One finds

$$
\Omega_{j}(z, a)=M\left(z-a_{j}+a_{j}^{0}, a^{0}\right)+\sum_{k} \frac{A_{k}\left(a^{0}\right)}{z-a_{j}+a_{j}^{0}-a_{k}^{0}},
$$


where it will be convenient to introduce the notation

$$
A_{k}\left(a^{0}\right)=(-1)^{k-1} \lambda C_{k}\left(a^{0}\right)
$$

One can use this to define a connection

$$
\nabla_{j}=d_{z}+d_{a}-\Omega_{j}(z, a) d\left(z-a_{j}\right)
$$

on the trivial bundle

$$
\left(D_{j} \times U \backslash \Delta_{j}\right) \times \mathbf{C}^{2} .
$$

Observe that $\epsilon$ has been chosen small enough so that the only singularities in $\Omega_{j}(z, a)$ for $z \in D_{j}$ occur when $z=a_{j}$ (where there is a simple pole). Let $\bar{D}$ denote the union of the closed disks $\bar{D}_{j}\left(\rho_{1, j}\right)$ for $j=1,2$. Now we extend $\nabla^{0}$ to a connection on the trivial bundle

$$
\mathbf{C} \backslash \bar{D} \times U \times \mathbf{C}^{2},
$$

in the following fashion

$$
\nabla_{\infty}=d_{z}+d_{a}-\Omega\left(z, a^{0}\right) d z
$$

Observe that $\nabla_{\infty}$ has no singularities on the domain we consider it on. Next we will show that the connections $\nabla_{j}$ and $\nabla_{\infty}$ are gauge equivalent to one another on the domain $\delta D_{j} \times U$ by a gauge transformation $S_{j}(z, a)$ which is normalized by the condition that $S_{j}\left(z, a^{0}\right)=I$ (the identity matrix). More specifically

$$
\nabla_{j}=S_{j}^{-1} \nabla_{\infty} S_{j}
$$

on $\delta D_{j} \times U$. To prove the existence of $S_{j}(z, a)$ we introduce parallel transport relative to the connection $\nabla_{j}$. If $\gamma$ is a smooth curve in $\delta D_{j} \times U$ then we write $P_{j}(\gamma)$ for parallel transport along the curve $\gamma$ relative to the connection $\nabla_{j}$. Since the bundle we consider is trivial we may think of $P_{j}(\gamma)$ as a linear map on $\mathbf{C}^{2}$. Now define

$$
\gamma_{j}(t, z, a)=\left(z,(1-t) a_{1}^{0}+t a_{1},(1-t) a_{2}^{0}+t a_{2}\right) \text { for } z \in D_{j}, a \in U \text {. }
$$

Then

$$
[0,1] \ni t \rightarrow \gamma(t, z, a)
$$

is a curve in $\delta D_{j} \times U$ which joins $\left(z, a^{0}\right)$ to $(z, a)$. We will denote this curve by $\gamma_{j}(z, a)$ and define

$$
S_{j}^{-1}(z, a)=P_{j}\left(\gamma_{j}(z, a)\right)
$$


Observe that we have defined the inverse $S_{j}^{-1}$ rather than $S_{j}$. Because the connection $\nabla_{j}$ is integrable we can replace $\gamma_{j}(z, a)$ by any smooth curve in $\delta D_{j} \times U$ which joins $\left(z, a^{0}\right)$ to $(z, a)$ but keeps the projection on the first coordinate (i.e., $\left.z\right)$ fixed. The matrix $S_{j}$ of parallel translation thus satisfies

$$
d_{a} S_{j}(z, a)=-S_{j}(z, a) \Omega_{j}(z, a) d a .
$$

Now suppose that $z \in \delta D_{j}$ and let $B_{j}(z)$ be a small ball about $z$ contained in the annulus $\delta D_{j}$. Suppose that $u \in B_{j}(z)$ and let,

$$
P_{j}(u, z \mid a)
$$

denote parallel translation with respect to $\nabla_{j}$ along any smooth curve that joins $(z, a)$ to $(u, a)$ whose projection on the first coordinate stays inside $B_{j}(z)$ and whose projection on the second coordinate is fixed at $a$. Again because the connection is integrable and the homotopy class of any such curve is fixed, parallel translation does not depend on the choice of such a curve and we have

$$
d_{u} P_{j}(u, z \mid a)=\Omega_{j}(u, a) P_{j}(u, z \mid a) d u .
$$

Once again using the fact that parallel translation depends only on the homotopy class of the path we can write

$$
S_{j}(u, a) P_{j}(u, z \mid a)=P_{j}\left(u, z \mid a^{0}\right) S_{j}(z, a) .
$$

Differentiating this last relation with respect to $u$ using (2.5) and then setting $u=z$ one finds

$$
d_{z} S_{j}(z, a)+S_{j}(z, a) \Omega_{j}(z, a) d z=\Omega_{j}\left(z, a^{0}\right) S_{j}(z, a) d z .
$$

But since $\Omega_{j}\left(z, a^{0}\right)=\Omega\left(z, a^{0}\right)$ we can combine (2.4) and (2.6) to get (2.3).

Now we wish to factor $S_{j}(z, a)$,

$$
S_{j}(z, a)=\Sigma_{\infty}(z, a)^{-1} \Sigma_{j}(z, a),
$$

where $\Sigma_{j}(z, a)$ is a holomorphic, invertible matrix valued function for $(z, a) \in D_{j} \times$ $U$, and $\Sigma_{\infty}(z, a)$ is a holomorphic invertible matrix valued function for $z$ outside the union of the disks $\bar{D}_{j}\left(\rho_{1, j}\right)$ and $a \in U$. We further require that $\Sigma_{\infty}(z, a)$ is normalized so that

$$
\Sigma_{\infty}(z, a)=I+O\left(z^{-1}\right)
$$

In Malgrange [7] it is shown that by choosing a sufficiently small neighborhood $U$ of $a^{0}$ this Wiener-Hopf factorization problem can be solved. Suppose now that $U$ is sufficiently small so (2.7) is valid. Combining (2.7) and (2.3) we find

$$
\Sigma_{j}^{-1} \nabla_{j} \Sigma_{j}=\Sigma_{\infty}^{-1} \nabla_{\infty} \Sigma_{\infty}
$$


over the set $\delta D_{j} \times U$ in the base. On the left hand side of (2.8) is a connection which extends to $D_{j} \times U \backslash \Delta_{j}$ with simple poles at $z=a_{j}$ and on the right hand side the connection extends to a connection on $\mathbf{C} \backslash \bar{D} \times U$. We denote by $\nabla$ the connection whose existence is thus assured by (2.8). Examining the singularity structure of this connection at $z=a_{j}$ and $z=\infty$ using the properties of $\Sigma_{j}$ and $\Sigma_{\infty}$ we find that,

$$
\nabla=d_{z}+d_{a}-M(z, a) d z-\sum_{k} \frac{A_{k}(a)}{z-a_{k}} d\left(z-a_{k}\right),
$$

where $M(z, a)$ is a polynomial in $z$ with the same leading terms as $M\left(z, a^{0}\right)$ and hence the same leading terms as $\Omega(z)$ near infinity, and $A_{k}(a)$ agrees with $A_{k}\left(a^{0}\right)$ at $a=a^{0}$. To make contact with the developments in $\S 1$ we introduce $C_{k}(a)$ by

$$
A_{k}(a)=(-1)^{k-1} \lambda C_{k}(a) .
$$

The distinguished fundamental solution for $\nabla$ whose monodromy is to remain fixed as a function of $a$ is given by,

$$
Y(z, a)=\Sigma_{\infty}^{-1}(z, a) Y\left(z, a^{0}\right),
$$

for $(z, a) \in \mathbf{C} \backslash \bar{D} \times U$. It is clear that this has exactly the same monodromy multiplier as $Y\left(z, a^{0}\right)$, as $z$ makes a circuit of either of the circles $\Gamma_{j}$.

Now we turn to the definition of the $\tau$-function. Let $H$ denote the Hilbert space,

$$
H=H^{\frac{1}{2}}\left(\Gamma_{1}\right) \oplus H^{\frac{1}{2}}\left(\Gamma_{2}\right),
$$

where $H^{\frac{1}{2}}$ denotes the Sobolev space of order $\frac{1}{2}$. Let $H_{+}$denote the subspace of $H$ which consists of boundary values of functions analytic in the open disks $D_{j}\left(r_{j}\right)$ for $j=1,2$ (recall that the circle $\Gamma_{j}$ is the boundary of the disk $D_{j}\left(r_{j}\right)$ ). Let $H_{-}$ denote the boundary values in $H$ of functions analytic in

$$
\mathbf{C} \backslash\left\{\bar{D}_{1}\left(r_{1}\right) \cup \bar{D}_{2}\left(r_{2}\right)\right\}
$$

which vanish at $z=\infty$. Then $H=H_{+} \oplus H_{-}$and we denote the projections relative to this decomposition by $P_{ \pm}$. If $S$ is a smooth matrix valued function defined on the circles $\Gamma_{j}$ for $j=1,2$ it defines a map

$$
S: H \rightarrow H
$$

acting by multiplication by $S$ in the obvious way. The Toeplitz operator, $T_{S}$, associated with $S$ is defined by

$$
T_{S}=P_{+} S P_{+} .
$$


We are now prepared to define the $\tau$ - function. Let $S$ denote the matrix valued function which is equal to $S_{j}$ on $\Gamma_{j}$ for $j=1,2$. Then, following Malgrange, we define

$$
d \log \tau=\operatorname{Tr}\left(T_{S}^{-1} T_{d_{a} S}-T_{S^{-1} d_{a} S}\right) .
$$

The inverse $T_{S}^{-1}$ is computable in terms of the factorization (2.7) by the standard Wiener-Hopf technique and (see lemma 6.7 in [7]) one finds

$$
d \log \tau=\frac{1}{2 \pi i} \sum_{j} \int_{\Gamma_{j}} \operatorname{Tr}\left(\Sigma_{j}^{-1} d_{z} \Sigma_{j} \wedge \Sigma_{\infty}^{-1} d_{a} \Sigma_{\infty}\right) .
$$

In [10] the $\tau$-function defined by (2.10) is shown to have the following significance. The family of subspaces

$$
W(a)=Y\left(\cdot, a^{0}\right)^{-1} S(\cdot, a) H_{+}
$$

determine boundary conditions for a Cauchy-Riemann operator localized in the exterior of $\bar{D}$. This Cauchy-Riemann operator has a domain which incorporates functions which have suitable branching at the points $a_{j}$. The subspaces $W(a)$ belong to a Grassmannian over which there sits a holomorphic line bundle det* This line bundle has a connection whose curvature vanishes over the family of subspaces $W(a)$. The $\tau$ - function defined by (2.10) is the ratio of the canonical section of det* to a section of det* that is flat with respect to this connection. It is also explained in [10] that this makes it possible to regard $\tau$ as the determinant of a singular Cauchy-Riemann operator. The reader is refered to [10] for details.

From (2.8) and (2.9) we see that since

$$
\Sigma_{\infty}^{-1} \nabla_{\infty} \Sigma_{\infty}=\nabla
$$

we have

$$
\Sigma_{\infty}^{-1}(z, a) d_{a} \Sigma_{\infty}(z, a)=-\sum_{k} \frac{A_{k}(a)}{z-a_{k}} d a_{k} .
$$

Using (2.8) and (2.9) and

$$
\Sigma_{j}^{-1} \nabla_{j} \Sigma_{j}=\nabla
$$

one sees that

$$
\Sigma_{j}^{-1}(z, a) d_{z} \Sigma_{j}(z, a)=\Omega(z, a) d z-\Sigma_{j}^{-1}(z, a) \Omega_{j}(z, a) \Sigma_{j}(z, a) d z,
$$

where

$$
\Omega(z, a)=M(z, a)+\sum_{k} \frac{A_{k}(a)}{z-a_{k}} .
$$


Since $\Sigma_{j}(z, a)$ is an invertible holomorphic function on $D_{j} \times U$ it follows that the pole type singularities in the two terms on the right hand side of (2.13) must cancel. One may easily confirm that this implies

$$
A_{j}(a)=\sigma_{j}^{-1} A_{j}\left(a^{0}\right) \sigma_{j}
$$

where

$$
\sigma_{j}:=\Sigma_{j}\left(a_{j}, a\right)
$$

We write

$$
\Sigma_{j}(z, a)=\sigma_{j}\left(I+\beta_{j}\left(z-a_{j}\right)\right)+O\left(\left(z-a_{j}\right)^{2}\right)
$$

for the first terms in the Taylor expansion of $\Sigma_{j}$ near $z=a_{j}$. Using (2.13) and (2.14) one finds that

$$
\Sigma_{j}^{-1} \partial_{z} \Sigma_{j}=B_{j}(a)-\sigma_{j}^{-1}\left(B_{j}\left(a^{0}\right)-\left[A_{j}\left(a^{0}\right), \beta_{j}\right]\right) \sigma_{j}+O\left(\left(z-a_{j}\right)\right),
$$

where

$$
B_{j}(a)=M\left(a_{j}, a\right)+\sum_{k \neq j} \frac{A_{k}(a)}{a_{j}-a_{k}} .
$$

Substituting (2.15) and (2.12) into the formula (2.11) one finds

$$
d_{a} \log \tau=\sum_{j} \operatorname{Tr}\left(B_{j}(a) A_{j}(a)-B_{j}\left(a^{0}\right) A_{j}\left(a^{0}\right)\right) d a_{j},
$$

where we made use the centrality of the trace, (2.14) again, and the fact that

$$
\operatorname{Tr}\left(\left[A_{j}\left(a^{0}\right), \beta_{j}\right] A_{j}\left(a^{0}\right)\right)=0 .
$$

If we use $A_{j}(a)=(-1)^{j-1} \lambda C_{j}(a)$ to compare (2.16) with (2.0) and (2.2) we find

$$
d_{a} \log \operatorname{det}(1-\lambda K)=\sum_{j} \operatorname{Tr}\left(B_{j}(a) A_{j}(a)\right) d a_{j},
$$

from which it follows that the $\tau$-function differs from $\operatorname{det}(1-\lambda K)$ by the exponential of a linear function of $a$.

\section{References}

[1] Beals, R., Sattinger, D.H. : Integrable systems and isomonodromy deformations, preprint, Yale University. 
[2] Harnad, J., Tracy, C.A., Widom, H. : Hamiltonian structure of equations appearing in random matrices, in Low dimensional topology and Quantum Field Theory, ed. H. Osborn, NATO ASI Series B, Vol. 314, Plenum Press (New York), 231-245 (1993).

[3] Its, A.R., Izergin, A.G., Korepin, V.E., Slavnov, N.A. : Differential equations for quantum correlation functions, Int. J. Mod. Physics B 4, 1003-1037 (1990).

[4] Jimbo, M., Miwa, T., Môri, Y., Sato, M. : Density matrix of an impenetrable Bose gas and the fifth Painlevé transcendent, Physica 1D, 80-158 (1980).

[5] Jimbo, M., Miwa, T., Ueno, K. : Monodromy preserving deformations of linear ordinary differential equations with rational coefficients I., Physica 2D, 306-352 (1981).

[6] Jimbo, M., Miwa, T. : Monodromy preserving deformations of linear ordinary differential equations with rational coefficients II, III., Physica 2D, 407-448 (1981); Physica 4D, 26-46 (1983).

[7] Malgrange, B. : Sur les deformations isomonodromiques, in Mathematique et Physique: Seminaire de l'Ecole Normale Superieure, 1979-1982 edited by L.B. de Monvel, A. Doudy, and J.L. Verdier, Birkhauser, Boston, 400-426 (1983).

[8] Mehta, M.L., : Random Matrices, $2^{\text {nd }}$ edition, Academic Press, San Diego.

[9] Moore, G. : Matrix models of 2D gravity and isomonodromic deformation, Prog. Theor. Physics Suppl. No. 102, 255-285 (1990).

[10] Moore, G. : Geometry of the string equations, Comm. Math. Phys. 133, 261-304 (1990).

[11] Palmer, J. : Determinants of Cauchy-Riemann operators as $\tau$-functions, Acta Applicandae Mathematicae 18 No. 3, 199-223 (1990).

[12] Tracy, C.A., Widom, H. : Introduction to random matrices, Geometric and Quantum Aspects of Integrable Systems, G. F. Helminck, Lecture Notes in Physics, Vol. 424, Springer-Verlag (Berlin), 103-130, (1993).

[13] Tracy, C.A., Widom, H. : Level spacing distributions and the Airy kernel, to appear in Commun. Math. Phys.

[14] Tracy, C.A., Widom, H. : Level spacing distributions and the Bessel kernel, to appear in Commun. Math. Phys.

[15] Tracy, C.A., Widom, H. : Fredholm determinants, differential equations and 
matrix models, to appear in Commun. Math. Phys. 